\title{
Cortical propagating waves: amplifying and suppressive?
}

\author{
Matteo di Volo ${ }^{1,2} \cdot$ Sandrine Chemla ${ }^{3} \cdot$ Alain Destexhe $^{1}$
}

Received: 18 March 2021 / Revised: 12 May 2021 / Accepted: 14 May 2021 / Published online: 18 May 2021

(c) The Author(s), under exclusive licence to Springer Science+Business Media, LLC, part of Springer Nature 2021

In this commentary, we would like to revisit a recent publication by Davis et al. (2020) showing that propagating waves in cerebral cortex could serve to boost the response to visual stimuli and gate perception. It seems therefore interesting to us to relate these observations to the suppressive traveling waves previously observed in the awake monkey (Chemla et al., 2019). We would like to overview these two results, clarifying that they are compatible and suggest that they may represent different facets of the same phenomenon. Consistent with this, we propose that the mechanism of suppressive waves (also called "suppression mechanism" thereafter) modulates the response to visual stimuli and, ultimately, their detection.

In detail, the experiments of Davis et al. (2020) were done using multi-electrode recordings (units and LFPs) in awake marmosets. It is well known that neuronal spiking rate is modulated by their phase relationship with the LFP oscillation. In their article they demonstrate that there is also a LFP phase alignment with the stimulus detection (for LFP measured at the retinotopic location of the stimulus to be detected). One of the remarkable aspects of their results is that this facilitation of detection (and of spiking rate) occurs only if this phase alignment is coming from an LFP transient generated by a spontaneous propagating LFP wave.

But what is the underlying mechanism that would specifically generate an activity and a perception increase when the LFP transient propagates? In a recent study (Chemla et al., 2019), it was shown that the interaction between propagating waves reveals a suppressive component in awake macaque V1. Indeed, in these experiments recorded in voltagesensitive dye (VSD) imaging, the "collision" between two

Communicated by Action Editor: Ken Miller.

\footnotetext{
Alain Destexhe

destexhe@unic.cnrs-gif.fr

Paris-Saclay University, CNRS, Gif-sur-Yvette, France

2 Paris-Cergy University, Cergy, France

3 Aix-Marseille University, CNRS, Marseille, France
}

propagating waves (generated by a spatio-temporal sequence of two local stimuli) was compared to the linear summation of each wave measured independently (linear prediction), and the combined waves were always found to be sub-linear (Chemla et al., 2019). This defines a suppressive component which, remarkably, also propagates as a wave. This suppressive wave is thus defined as the nonlinearity resulting from the difference between the evoked activity and the linear prediction. The mechanisms underlying the suppressive wave were found to be the difference in gain control between excitatory and inhibitory neurons and conductance-based interactions.

Speaking of suppressive waves in macaque V1 may appear to be at odds with the positive modulatory effects of the waves found by Davis et al. in marmoset. However, even if the response to the two stimuli sequence is measured to be sublinear in Chemla et al., it is perfectly compatible with an augmentation of activity at the arrival of the second wave, therefore in accordance with the results of Davis et al. Our hypothesis is that, even if Davis et al. considered spontaneous waves (while the suppression measured in Chemla et al. (2019) is found between the evoked responses to two visual stimuli), the two phenomena are consistent and could underline the same cellular mechanism.

It is important to point that Davis et al. did not measure suppression, because their analysis at the single-trial level cannot be easily used to compare a linear prediction with the observation. Therefore it is possible that also in this case neurons' activity increases sublinearly at the arrival of the stimuli. As a result the amount of such increase will be crucially dependent on the suppression mechanism described in Chemla et al.. In their results, Davis et al. found an increased firing in the recorded units for a particular phase of the LFP traveling wave. Such an increase of firing may affect differently excitatory (E) and inhibitory (I) cells. Since I cells have higher gain, they are expected to increase more than $\mathrm{E}$ cells, which was found to be one of the main mechanisms of the observed suppressive waves (Chemla et al., 2019). This is also compatible with paradoxical effects observed in the stabilized supralinear network (Ozeki et al., 2009; Rubin 
et al., 2015; Tsodyks et al., 1997). In Davis et al. cells were not discriminated, so the increased firing could result from different combinations of $\mathrm{E}$ and I cells, yielding a net facilitatory or suppressive wave at the population level (in terms of nonlinear summation). So, in theory, the increased unit activity could be compatible with the suppressive waves observed in Chemla et al.. Also note that the LFP has been found to primarily reflect the activity of FS cells in human cortex (Telenczuk et al., 2017), which reinforces the possibility that there is a significant inhibitory component in the propagating waves observed in the LFP.

We simulated this situation using a computational model. In Fig. 1 we show that a suppressive collision between waves is compatible with an increase in firing activity at the input location. Consistently with the results from Fig. 4a of Davis et al. (2020), such an increase depends on the wave phase at which the stimulus arrives. Notice that here, inhibitory neurons have a much larger increase in firing activity with respect to excitatory cells, which constitutes experimentally testable evidence for suppressive waves.

Thus, mechanistically, the results of Davis et al. are compatible with suppressive waves, but are suppressive waves compatible with perception? We propose that, similar to the effect of disambiguating apparent motion representation in V1 maps, the LFP wave could reflect a dynamic suppression propagating out of the feedforward input evoked by the visual stimulus. Indeed, the mechanism shown in Chemla et al. (2019) suggests that a suppressive wave could be expected to result from the interaction between the spontaneous wave and the wave evoked by the stimulus. The non-linearity of this interaction can be a very effective mechanism to shape the activity at the position of the stimulus representation by dynamically improving the signal-to-noise ratio and hereby increase the detectability and therefore the perception of

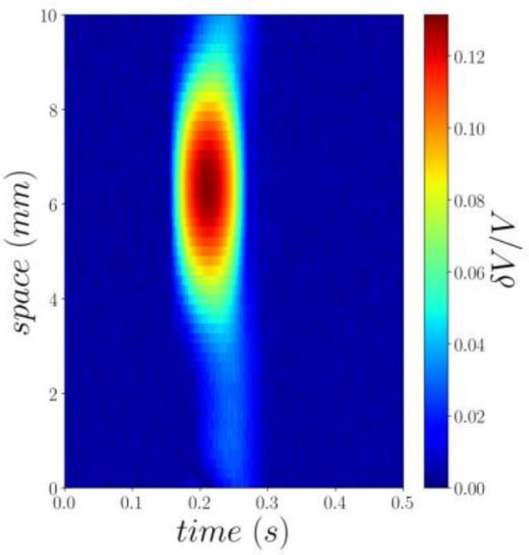

d

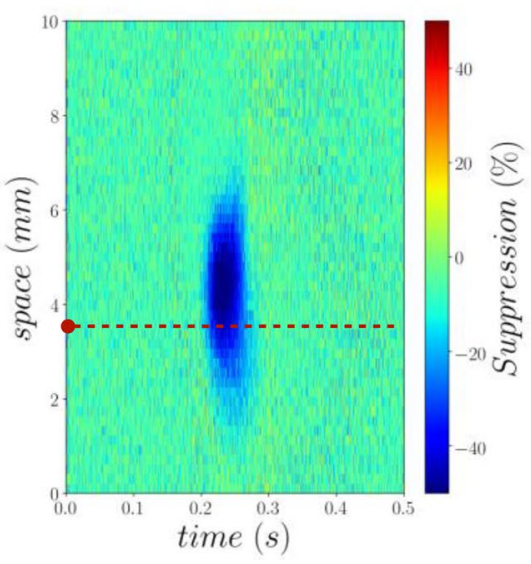

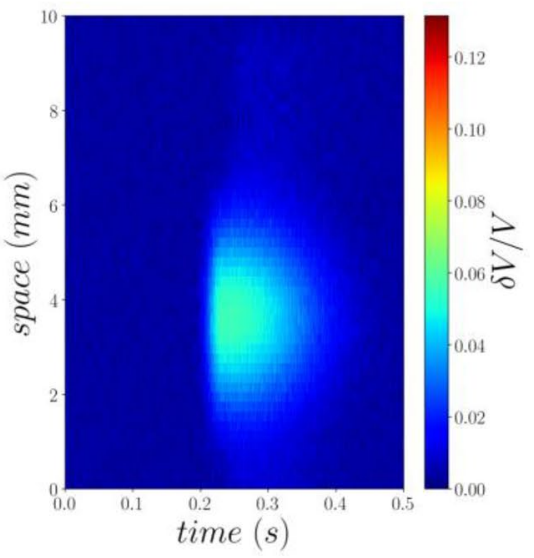

e

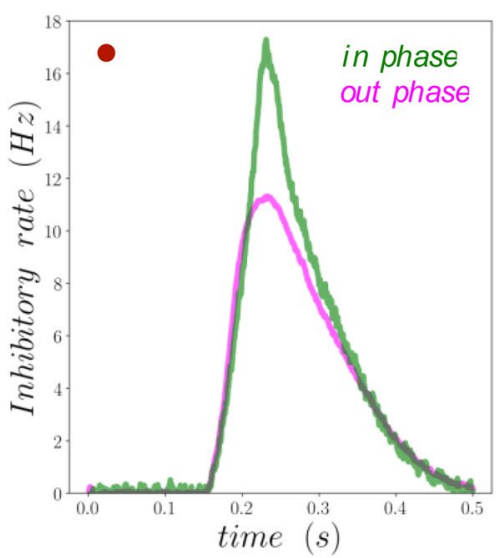

c Wave+ Stimulus

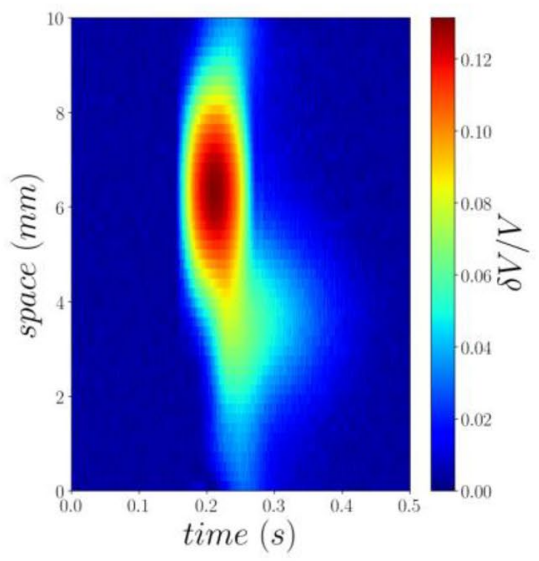

f

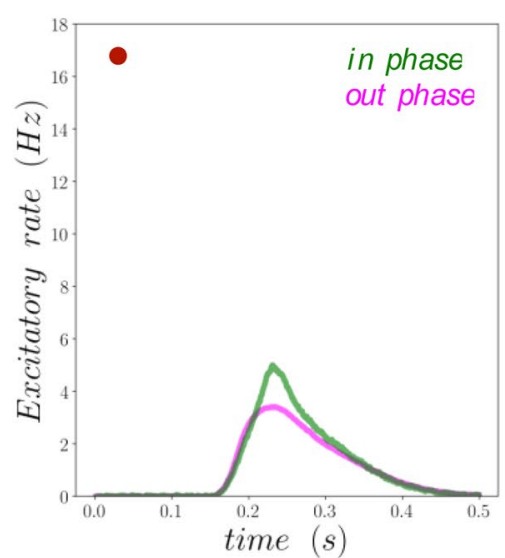

Fig. 1 Computational model of wave-stimulus interaction. a Spatio-temporal profile of the wave. b Spatio-temporal profile of the response to the stimulation. c Spatio-temporal profile of the response to the stimulation with the wave. d Spatio-temporal profile of the suppressive wave, estimated by subtracting the activiy measured in $\mathbf{c}$ minus the sum of the activity in $\mathbf{a}$ and in $\mathbf{b}$. e Change in firing rate of inhibitory cells at the input location (red line in panel d). Green curve is obtained for stimuli arriving in phase with the wave while magenta for out of phase inputs. f Same as e for excitatory cells. Details on model and parameters in Chemla et al. (2019) 
the stimulus. Notice that a sublinear increase in the activity at the center of the stimuli will still increase neural firing with respect to a situation in absence of spontaneous waves.

In conclusion, we discussed here how suppressive waves (Chemla et al., 2019) could contribute to the result presented in Davis et al. (2020). Such suppressive interactions were shown to provide the visual system with a simple mechanism to disambiguate ambiguous visual stimuli, providing a new functional role for propagating waves in awake monkey (Chemla et al., 2019). In support of this, a recent study found that there is a similar suppression between propagating waves in mouse S1 (di Volo \& Ferezou, 2021). Davis et al. have shown that waves are a necessary neuronal spatio-temporal pattern to increase neuronal response and detectability of the stimulus - this amplification therefore participates directly to perception. We proposed here that waves could gate perception and at the same time, be accompanied by a suppressive wave that would increase the signal-to-noise of the stimulus representation. This suggests that all these cortical waves could stem from the same underlying mechanisms.

Acknowledgements We thank Frederic Chavane for many stimulating discussions. Research supported by the CNRS, the ANR (ShootingStar project) and the European Community (Human Brain Project, H2020-945539).

\section{Declarations}

Conflict of interest The authors declare that they have no conflict of interest.

\section{References}

Chemla, S., et al. (2019). Suppressive traveling waves shape representations of illusory motion in primary visual cortex of awake primate. Journal of Neuroscience. https://doi.org/10.1523/JNEUROSCI. 2792-18.2019

Davis, Z. W., Muller, L., Martinez-Trujillo, J., Sejnowski, T., \& Reynolds, J. H. (2020). Spontaneous travelling cortical waves gate perception in behaving primates. Nature. https://doi.org/10. 1038/s41586-020-2802-y

Ozeki, H., Finn, I. M., Schaffer, E. S., Miller, K. D., \& Ferster, D. (2009). Inhibitory Stabilization of the Cortical Network Underlies Visual Surround Suppression. Neuron. https://doi.org/10.1016/j. neuron.2009.03.028

Rubin, D. B., VanHooser, S. D., \& Miller, K. D. (2015). The stabilized supralinear network: A unifying circuit motif underlying multiinput integration in sensory cortex. Neuron. https://doi.org/10. 1016/j.neuroN.2014.12.026

Telenczuk, B., et al. (2017). Local field potentials primarily reflect inhibitory neuron activity in human and monkey cortex. Science and Reports. https://doi.org/10.1038/srep40211

Tsodyks, M. V., Skaggs, W. E., Sejnowski, T. J., \& McNaughton, B. L. (1997). Paradoxical effects of external modulation of inhibitory interneurons. Journal of Neuroscience. https://doi.org/10.1523/ jneurosci.17-11-04382.1997

di Volo, M. \& Ferezou, I. (2021). Nonlinear collision between anisotropic propagating waves in mouse somatosensory cortex. bioRxiv.

Publisher's Note Springer Nature remains neutral with regard to jurisdictional claims in published maps and institutional affiliations. 